\title{
Nonlinear impairments in mode division multiplexed systems
}

\author{
Kutluyarov, Ruslan V.; Lyubopytov, Vladimir S.; Bagmanov, Valeriy Kh; Sultanov, Albert Kh
}

Published in:

Proceedings of SPIE

Link to article, DOI:

$10.1117 / 12.2318875$

Publication date:

2018

Document Version

Publisher's PDF, also known as Version of record

Link back to DTU Orbit

Citation (APA):

Kutluyarov, R. V., Lyubopytov, V. S., Bagmanov, V. K., \& Sultanov, A. K. (2018). Nonlinear impairments in mode division multiplexed systems. In Proceedings of SPIE (Vol. 10774). [107741U] SPIE - International Society for Optical Engineering. Proceedings of SPIE - The International Society for Optical Engineering Vol. 10774 https://doi.org/10.1117/12.2318875

\section{General rights}

Copyright and moral rights for the publications made accessible in the public portal are retained by the authors and/or other copyright owners and it is a condition of accessing publications that users recognise and abide by the legal requirements associated with these rights.

- Users may download and print one copy of any publication from the public portal for the purpose of private study or research.

- You may not further distribute the material or use it for any profit-making activity or commercial gain

- You may freely distribute the URL identifying the publication in the public portal 


\section{Nonlinear impairments in mode division multiplexed systems}

Ruslan V. Kutluyarov, Vladimir S. Lyubopytov, Valeriy Kh. Bagmanov, Albert Kh. Sultanov

Ruslan V. Kutluyarov, Vladimir S. Lyubopytov, Valeriy Kh. Bagmanov, Albert Kh. Sultanov, "Nonlinear impairments in mode division multiplexed systems," Proc. SPIE 10774, Optical Technologies in Telecommunications 2017, 107741U (6 June 2018); doi: 10.1117/12.2318875 


\title{
Nonlinear impairments in mode division multiplexed systems
}

\author{
Ruslan V. Kutluyarov*a, Vladimir S. Lyubopytov ${ }^{\mathrm{a}, \mathrm{b}}$, Valeriy Kh. Bagmanov ${ }^{\mathrm{a}}$, Albert Kh. Sultanov ${ }^{\mathrm{a}}$ \\ ${ }^{a}$ Ufa State Aviation Technical University, 12 K. Marx St., Ufa, 450000, Russia; ${ }^{\mathrm{b}}$ Technical \\ University of Denmark (DTU), Ørsteds Plads 343, Kgs. Lyngby, 2800, Denmark
}

\begin{abstract}
In this paper we consider nonlinear impairments of mode division multiplexed signals with QAM modulation in optical fibers with linear mode coupling of spatial modes. We simulate simultaneous propagation of fundamental mode and two first-order vortex modes in standard single mode fiber at $850 \mathrm{~nm}$ and propagation of fundamental mode and first- and second-order vortices in step-index fiber with enlarged core at $1550 \mathrm{~nm}$. Simulation results shows that in strong coupling regime linear coupling lead to sufficient increasing of nonlinear impairments, but QAM-modulated signal is more robust to this effect than OOK modulated signal.
\end{abstract}

Keywords: vortex modes; few-mode fibers; Kerr-nonlinearities; mode division multiplexing

\section{INTRODUCTION}

Space division multiplexed (SDM) transmission on few-mode and multi-core optical fibers attracts enormous attention in recent years as a technology that may sufficiently increase capacity of fiber communications [1-4]. SDM fiber communications are challenging in many fields. One of the fundamental tasks is the choosing of proper waveguide. Communication with mode multiplexing may be developed on multi-core fiber with weak coupling between different cores [5] or on the fiber with one core. It was reported previously about special types of fibers with one core proposing very weak coupling between different spatial modes [6-8]. Another approach is based on the fibers with conventional step-index refractive profile but enlarged core. Such fibers provide propagation of finite number of spatial modes but modes are experienced by strong linear coupling [9]. One of the main advantages of this kind of fibers is the compatibility with conventional manufacturing process. Compensation of the linear mode coupling is another challenging task. It may be implemented by means of MIMO-DSP $[10,11]$ or by using of all-optical methods [12]. The first approach is very exacting for computational output of the receiver, and complexity of DSP is increasing very fast with number of modes. The second approach has great scalability but its commercial implementation require novel techniques for integrated devices performing optical signal processing. In any case, signal processing in electrical or optical domain is linear and any nonlinear impairments will sufficiently decrease the efficiency of compensation process. In general, nonlinearities are the fundamental reason that limits capacity of SDM communications. Therefore a lot of recent publications are devoted to numerical and theoretical analysis of nonlinear propagation of few spatial modes in quartz fibers. Set of generalized coupled nonlinear Schrödinger equations was derived in [13]. Following the approach developed for single-mode communications, the averaged Manakov-type equation was derived for multi-mode propagation in long haul communication lines [14, 15].

Recently theoretical and numerical methods for estimation of inter-channel nonlinear interference noise (NLIN) in multichannel WDM systems was developed. As shown in [15], the nonlinear interference in SDM systems is strongly influenced by the mechanisms that are responsible for linear mode coupling. Strong mode mixing implies a considerable reduction of the inter-channel NLIN power. In [16] it was shown that the presence of modal dispersion sufficiently decreases inter-modes nonlinear distortions. Modal dispersion between non-degenerate groups of modes leads to relative shifting of bit sequences in modal channels and mitigate its interaction. Similar effect was described for inter-channel nonlinear interaction via cross-phase modulation (XPM) for single-mode WDM-systems, when chromatic dispersion leads to «walk-off effect» for bit sequences of different spectral channels [17].

*kutluyarov.rv@net.ugatu.su; phone+7 347 273-0689; fax +7 347 273-0689

Optical Technologies for Telecommunications 2017, edited by Vladimir A. Andreev, Anton V. Bourdine,

Vladimir A. Burdin, Oleg G. Morozov, Albert H. Sultanov, Proc. of SPIE Vol. 10774, 107741U

(C) 2018 SPIE · CCC code: 0277-786X/18/\$18 - doi: 10.1117/12.2318875 
Previously we have studied numerically nonlinear mode coupling within the same spectral channel during simultaneous propagation of NRZ-coded modal channels $[18,19]$. It was shown that in the presence of linear mode coupling due to spontaneous power exchange between degenerate modes nonlinear impairments caused by XPM and self-phase modulation (SPM) are accumulated intensively. In this paper we evolve this approach and study nonlinear impairments for coherent modulation formats.

In this paper we do not consider propagation of polarization modes of the same spatial field distribution. The real case that corresponds to this assumption is the polarization maintaining fiber. Nevertheless, strict consideration of simultaneous polarization mode coupling and spatial mode coupling is the problem that requires a dedicated effort, which is left for the future research. Previously it was reported that interaction between polarization mode dispersion and Kerr-nonlinearities may lead to mitigation of nonlinear impairments, so simultaneous influence of polarization effects and spatial modes coupling effects on the nonlinear signal distortions is the very complex process.

Following the approach used in $[18,19]$ in this paper we study propagation of vortex modes, i.e. modes with helicoidal phase fronts.

\section{THEORETICAL BACKGROUND}

Considering simultaneous propagation of $N$ spatial modes of the same polarization one can define electromagnetic wave in frequency domain as

$$
\tilde{E}(r, \phi, z, \omega)=\sum_{p=1}^{N} \exp \left(i \beta_{p}(\omega) z\right) \tilde{A}_{p}(z, \omega) F_{p}(r) \exp \left(i m_{p} \phi\right),
$$

where $p$ is the mode number, $\beta_{p}$ is the propagation constant of the $p$-th spatial mode, $F_{p}(r, \phi)=F_{p}(r) \exp \left(\operatorname{im}_{p} \phi\right)$ is the transverse distribution of the $p$-th mode field, $m_{p}$ is the integer number defining azimuthal order of the $p$-th mode and $A_{p}$ is the slowly varying amplitude of the $p$-th mode.

Following the well-known approach one can derive a set of generalized coupled nonlinear Schrödinger equations [14]:

$$
\frac{\partial A_{p}}{\partial z}=i\left(\beta_{0 p}-\beta_{r}\right) A_{p}-\left(\beta_{1 p}-\frac{1}{v_{g r}}\right) \frac{\partial A_{p}}{\partial t}-i \frac{\beta_{2 p}}{2} \frac{\partial^{2} A_{p}}{\partial t^{2}}-\frac{\alpha}{2} A_{p}+i \sum_{l m n} f_{l m n p} \gamma A_{l}^{*} A_{m} A_{n}+i \sum_{m} q_{m p} A_{m},
$$

where $A_{p}$ is the slowly varying envelope expressed in a reference moving frame at a group velocity $v_{g r}, \beta_{r}$ is the reference propagation constant and $\gamma$ is the nonlinear parameter.

$$
\begin{gathered}
\beta_{0 p}=\beta_{p}\left(\omega_{0}\right), \\
\beta_{1 p}=\left.\frac{\partial \beta_{p}}{\partial \omega}\right|_{\omega_{0}}, \\
\beta_{2 p}=\left.\frac{\partial^{2} \beta_{p}}{\partial \omega^{2}}\right|_{\omega_{0}} .
\end{gathered}
$$

are respectively the propagation constant, inverse group velocity and group-velocity dispersion (GVD) of the $p$-th spatial mode.

Linear mode coupling in Equation 2 is defined by the coefficients

$$
q_{m p}(z)=\frac{k_{0}}{2 n_{e f f}\left(I_{m} I_{p}\right)^{1 / 2}} \iint \Delta n^{2}(x, y, z) F_{m} F_{p} d x d y,
$$

where the intensity normalization terms are defined as 


$$
I_{m}=\bar{n}_{m} / \bar{n}_{e f f} \iint F_{m}^{2}(x, y) d x d y .
$$

Here $n_{\text {eff }}$ and $n_{m}$ are the effective refractive indices for the fundamental mode and for the $m$-th mode respectively.

Linear coupling terms appear as a result of the perturbation of the refractive index $\Delta n^{2}(x, y, z)$ that may be caused by different mechanical stresses or by imperfections of the manufacturing process.

For simulation purposes fiber is derived as a concatenation of coaxial sections with constant parameters. Previously it was shown that fiber bending is the main reason of mode coupling [20]. Corresponding coupling coefficients are derived as

$$
q_{\mu \nu}^{(k)}=\frac{\gamma_{\mu} \gamma_{\nu}}{2 n_{1} k_{0} a \sqrt{e_{p} e_{m}}} \cdot \frac{\xi^{(k)}\left(\delta_{p, m-1}+\delta_{p, m+1}+\delta_{p, 1-m}\right)}{\left(\beta_{\mu}-\beta_{v}\right)^{2}} \Upsilon_{\mu \nu},
$$

where $n_{1}$ is the refractive index of the fiber core in the point $z=0, \xi(k)$ is the curvature of the $k$-th section, $p$ and $m$ are the mode azimuthal indices, $\delta$ is the Kronecker delta function, $e_{m}=2$ for $m=0$ and $e_{m}=1$ in other cases,

$$
\Upsilon_{\mu \nu}=\frac{J_{p}\left(\kappa_{\mu} a\right) J_{m}\left(\kappa_{v} a\right)}{\sqrt{\left|J_{p-1}\left(\kappa_{\mu} a\right) J_{p+1}\left(\kappa_{\mu} a\right) J_{m-1}\left(\kappa_{v} a\right) J_{m+1}\left(\kappa_{v} a\right)\right|}} .
$$

Here $J_{i}\left(\kappa_{\mu} a\right)$ is the Bessel function with $\kappa_{\mu}$ defined as

$$
\kappa_{\mu}=\sqrt{\left(k_{0} n_{1}\right)^{2}-\beta_{\mu}^{2}} .
$$

Nonlinear interaction of propagating modes is defined by set of coefficients derived by

$$
f_{l m n p}=\frac{A_{e f f}}{\left(I_{l} I_{\mathrm{m}} I_{n} I_{p}\right)^{1 / 2}} \iint F_{l}^{*} F_{m} F_{n} F_{p}^{*} d x d y,
$$

where $A_{\text {eff }}$ is the effective mode area [21].

Nonlinear impact on the propagated signal is derived by nonlinear operator in equation (2):

$$
\hat{N}=\sum_{l m n} f_{l m n p} \gamma A_{l}^{*} A_{m} A_{n} .
$$

Let consider the simplest case of nonlinear propagation, when three spatial modes are propagated through the fiber. Nonlinear operator (12) impacting on the third mode channel will be written as

$$
\hat{N}=f_{1223} A_{1}^{*} A_{2}^{2}+\left(f_{1313}+f_{1133}\right)\left|A_{1}\right|^{2} A_{3}+\left(f_{2323}+f_{2233}\right)\left|A_{2}\right|^{2} A_{3}+f_{3333}\left|A_{3}\right|^{2} A_{3},
$$

where the indices 1, 2, 3 correspond to the modes LP11-, LP01, LP11+ respectively.

The first term of the right-hand side of (13) corresponds to the four-wave mixing process that is negligible because of phase mismatch. The fourth term corresponds to the self-phase modulation. The second term corresponds to the XPM between conjugated first-order modes; the third term corresponds to the XPM between fundamental mode and the first order mode of interest. XPM efficiency depends on the difference between group velocities of interacting modes. In stepindex few modes fibers group velocities of modes with various orders are sufficiently different. Therefore efficiency of XPM process described by the second term is higher than the other one.

Note that linear mode coupling efficiency decreases with enlarging of the difference between group velocities. Therefore for qualitative analysis we assume that there is no power exchange between first-order modes and fundamental mode and XPM between these modes are negligible. If initial power of each mode channel is $P_{0}$ and power loss are negligible than total power of first-order modes remain constant and equal $2 P_{0}$.

Denote

$$
f_{1313}=f_{1133}=f_{3333}=f
$$


Using (14) one can define absolute value of nonlinear operator (13) as

$$
|\hat{N}|=4 f P_{0}\left|A_{3}\right|-f\left|A_{3}\right|^{3} .
$$

Function (15) has local maximum when $\left|A_{3}\right|=\sqrt{ }\left(4 P_{0} / 3\right)$. The physical meaning of this result is clear: the most significant distortions caused by Kerr-nonlinearities appear when the channel power increases due to the linear mode coupling.

\section{SIMULATION RESULTS}

It is well known that standard single mode fiber provides propagation of three spatial modes in the wavelength region near $850 \mathrm{~nm}$. Therefore we consider propagation of fundamental mode LP01 and conjugate first-order vortices LP11+ and LP11-. Basis of propagated modes defines the set of nonlinear coupling coefficients, calculated with (11). For three modes mentioned above we have 18 non-zero values [18]. But if we consider fiber that provides propagation of five spatial modes than we have 83 non-zero coefficients [19], see Table 1.

Table 1. Non-zero nonlinear coupling coefficients for a few-mode fiber with 5 spatial modes at $1550 \mathrm{~nm}$ (absolute values)

\begin{tabular}{|c|c|}
\hline LP mode indices & $f_{\text {Imnp }}$ \\
\hline LP01,LP01,LP01,LP01 & 1,0000 \\
\hline $\begin{array}{l}\text { LP11-, LP11-, LP11-, LP11-; LP11+, LP11+, LP11-, LP11-; LP11+, LP11-, LP11+, LP11-; } \\
\text { LP11-, LP11+, LP11-, LP11+; LP11-, LP11-, LP11+, LP11+; LP11+, LP11+, LP11+, LP11+ }\end{array}$ & 0,6965 \\
\hline $\begin{array}{l}\text { LP01, LP01, LP11-, LP11-; LP01, LP11-, LP01, LP11-; LP11+, LP01, LP01, LP11-; LP11-, LP01, } \\
\text { LP11-, LP01; LP01, LP11+, LP11-, LP01; LP11-, LP11-, LP01, LP01; LP11+, LP11+, LP01, LP01; } \\
\text { LP11-, LP01, LP01, LP11+; LP01, LP11+, LP01, LP11+; LP01, LP01, LP11+, LP11+ }\end{array}$ & 0,6589 \\
\hline $\begin{array}{l}\text { LP21-, LP21-, LP21-, LP21-; LP21+, LP21+, LP21-, LP21-; LP21+, LP21-, LP21+, LP21-; LP21- } \\
\text {, LP21+, LP21-, LP21+; LP21-, LP21-, LP21+, LP21+; LP21+, LP21+, LP21+, LP21+ }\end{array}$ & 0,6096 \\
\hline $\begin{array}{l}\text { LP11-, LP11-, LP21-, LP21-; LP11+, LP11+, LP21-, LP21-; LP11-, LP21-, LP11-, LP21-; } \\
\text { LP21+, LP11+, LP11-, LP21-; LP11+, LP21-, LP11+, LP21-; LP21+, LP11-, LP11+, LP21-; } \\
\text { LP21-, LP11-, LP21-, LP11-; LP11+, LP21+, LP21-, LP11-; LP21-, LP21-, LP11-, LP11-; } \\
\text { LP21+, LP21+, LP11-, LP11-; LP11+, LP21-, LP21+, LP11-; LP21+, LP11-, LP21+, LP11-; } \\
\text { LP11+, LP21-, LP21-, LP11+; LP11-, LP21+, LP21-, LP11+; LP21-, LP21-, LP11+, LP11+; } \\
\text { LP21+, LP21+, LP11+, LP11+; LP11-, LP21-, LP21+, LP11+; LP21+, LP11+, LP21+, LP11+; } \\
\text { LP21-, LP11+, LP11-, LP21+; LP11-, LP21+, LP11-, LP21+; LP21-, LP11-, LP11+, LP21+; } \\
\text { LP11+, LP21+, LP11+, LP21+; LP11-, LP11-, LP21+, LP21+; LP11+, LP11+, LP21+, LP21+ }\end{array}$ & 0,6035 \\
\hline $\begin{array}{l}\text { LP01, LP11-, LP11-, LP21-; LP11+, LP01, LP11-, LP21-; LP11+, LP11-, LP01, LP21-; LP11-, } \\
\text { LP01, LP21-, LP11-; LP01, LP11+, LP21-, LP11-; LP11-, LP21-, LP01, LP11-; LP21+, LP11+, } \\
\text { LP01, LP11-; LP01, LP21-, LP11+, LP11-; LP21+, LP01, LP11+, LP11-; LP11-, LP11+, LP21-, } \\
\text { LP01; LP21-, LP11-, LP11-, LP01; LP11+, LP21+, LP11-, LP01; LP11-, LP21-, LP11+, LP01; } \\
\text { LP21+, LP11+, LP11+, LP01; LP11+, LP11-, LP21+, LP01; LP21-, LP01, LP11-, LP11+; LP01, } \\
\text { LP21+, LP11-, LP11+; LP11-, LP21-, LP01, LP11+; LP11+, LP21+, LP01, LP11+; LP01, LP11-, } \\
\text { LP21+, LP11+; LP11+, LP01, LP21+, LP11+; LP11-, LP11+, LP01, LP21+; LP11-, LP01, LP11,, } \\
\text { LP21+; LP01, LP11+, LP11+, LP21+ }\end{array}$ & 0,5567 \\
\hline $\begin{array}{l}\text { LP01, LP01, LP21-, LP21-; LP01, LP21-, LP01, LP21-; LP21+, LP01, LP01, LP21-; LP21-, LP01, } \\
\text { LP21-, LP01; LP01, LP21+, LP21-, LP01; LP21-, LP21-, LP01, LP01; LP21+, LP21+, LP01, LP01; } \\
\text { LP21-, LP01, LP21+, LP01; LP21+, LP01, LP21+, LP01; LP21-, LP01, LP01, LP21+; LP01, LP21+, } \\
\text { LP01, LP21+; LP01, LP01, LP21+, LP21+ }\end{array}$ & 0,4514 \\
\hline
\end{tabular}

In this work we have simulated symmetrical single-polarization IQ-modulator as E/O converter in the transmitting side. Driving electrical signals are NRZ-coded with pseudo-random bit sequence and raised cosine filtered. Filter bandwidth was assumed equal $15 \mathrm{GHz}$ and rolloff factor was assumed equal 0.7 . 
We solve equations (2) numerically using Split-step Fourier method. For estimation of the nonlinear impairments, (2) were solved twice for each set of random parameters defining sections of simulated fiber: taking into account nonlinear terms of equations, and leaving them out of account. Thus, two envelopes of the field were found using this method. First of them takes into account both dispersion and nonlinear equation terms, while the second one takes into account only dispersion terms. Then rms variance of these two envelopes was calculated. For estimation of linear coupling influence we solved (2) in the presence of linear mode coupling and in the absence of it and then compared results.

In the first experiment series we have simulated propagation of LP11-, LP01, LP11+ mode channels with QAM-16 through 2.5-km SSMF at $850 \mathrm{~nm}$. Here and later QAM-symbol duration was equal $100 \mathrm{ps.} \mathrm{During} \mathrm{simulation} \mathrm{we} \mathrm{have}$ assumed mean length of coupling sections equal $25 \mathrm{~cm}$ that corresponds to strong mode coupling.

Results of the simulation are presented in Figure 1.

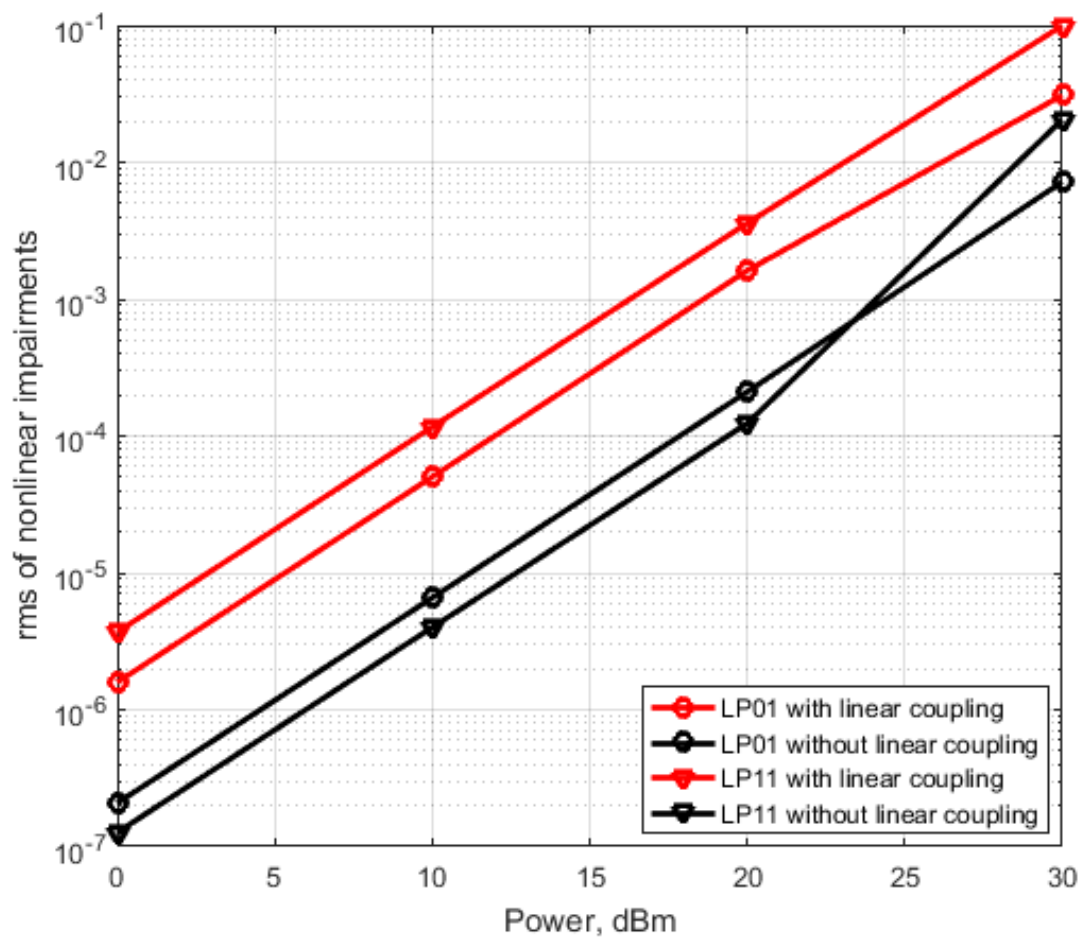

Figure 1. Nonlinear distortions in QAM-16 mode channels of SSMF in strong-coupling regime with $25 \mathrm{~cm}$ mean length coupling sections at $850 \mathrm{~nm}$

It is evident that the presence of linear coupling leads to sufficient increasing of nonlinear impairments for all the spatial modes.

In the second experiment series we have simulated propagation of LP21-, LP11-, LP01, LP11+, LP21+ mode channels at the $1550 \mathrm{~nm}$ with QAM-16 and QAM-64 modulation through 2.5-km fiber with enlarged core cross-section $(8.6 \mu \mathrm{m})$ and step-index refractive index profile. Additionally we have simulated weak mode coupling with mean length of fiber sections equal $5 \mathrm{~m}$.

Results of the simulation are presented in Figures 2 and 3. 
a)

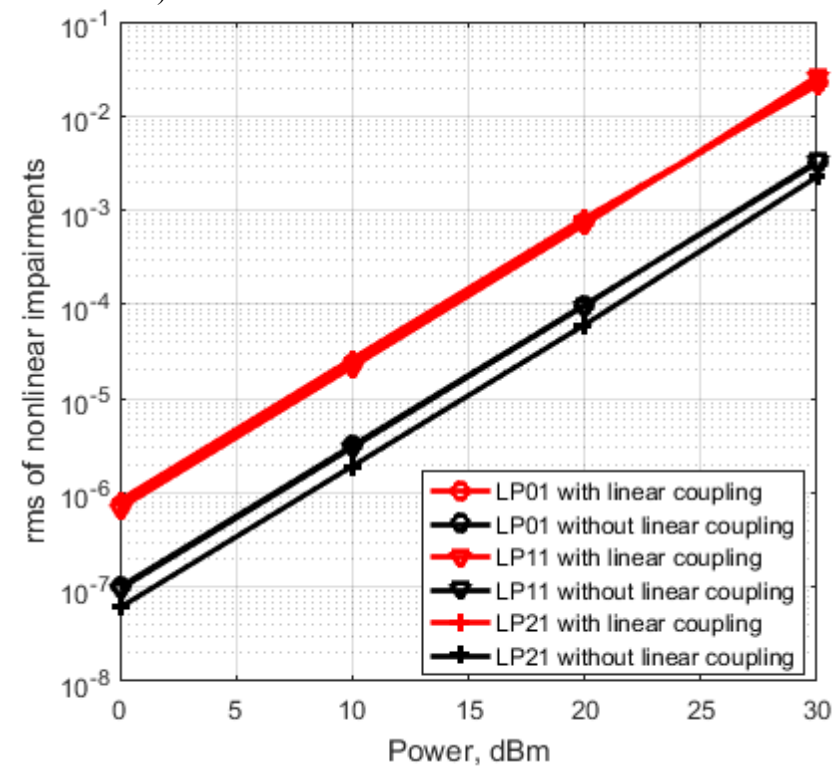

b)

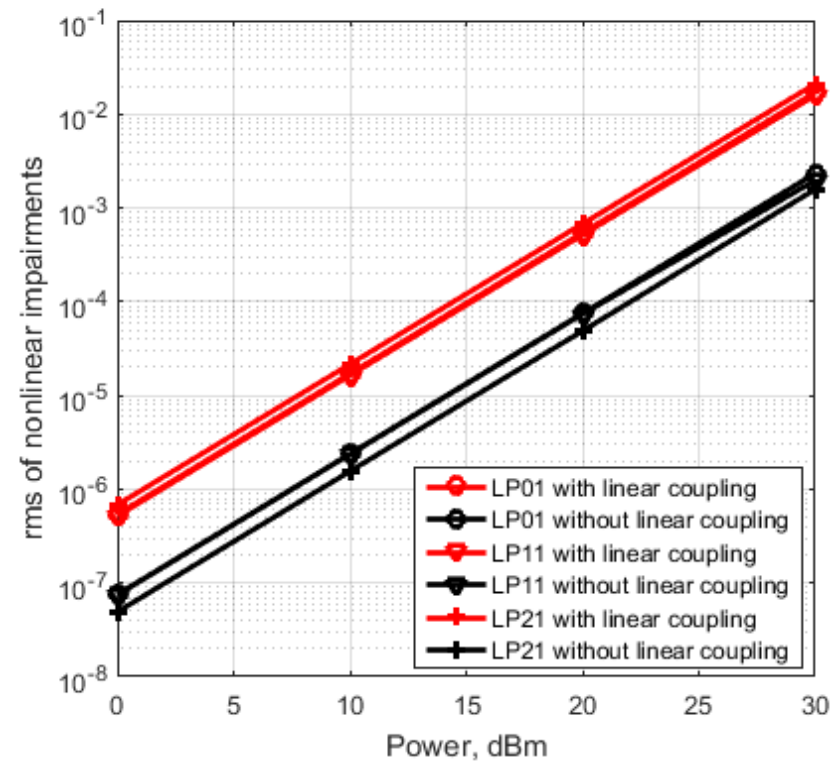

Figure 2. Nonlinear distortions in mode channels in strong-coupling regime with $25 \mathrm{~cm}$ mean length coupling sections at $1550 \mathrm{~nm}$. a) QAM-16; b) QAM-64.

a)

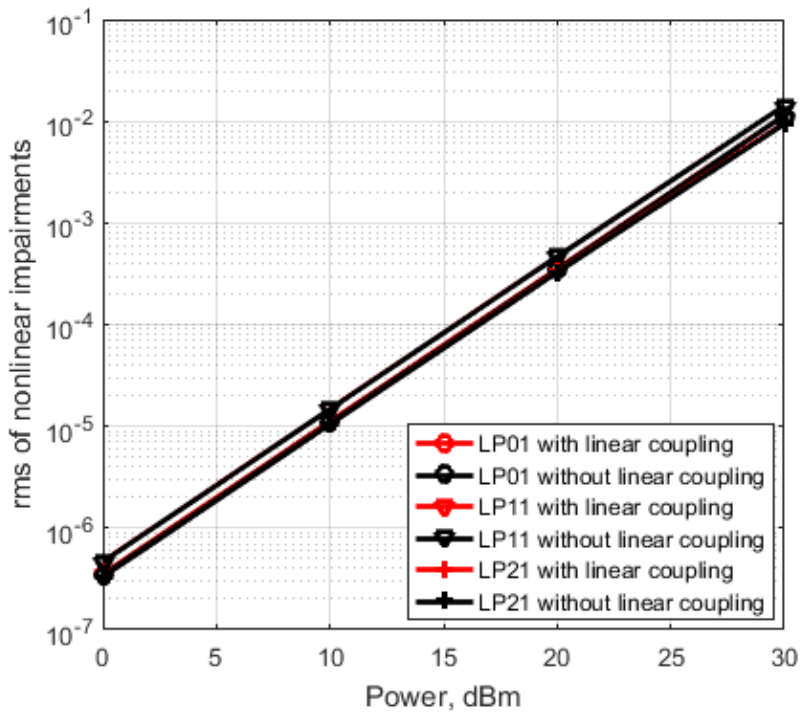

b)

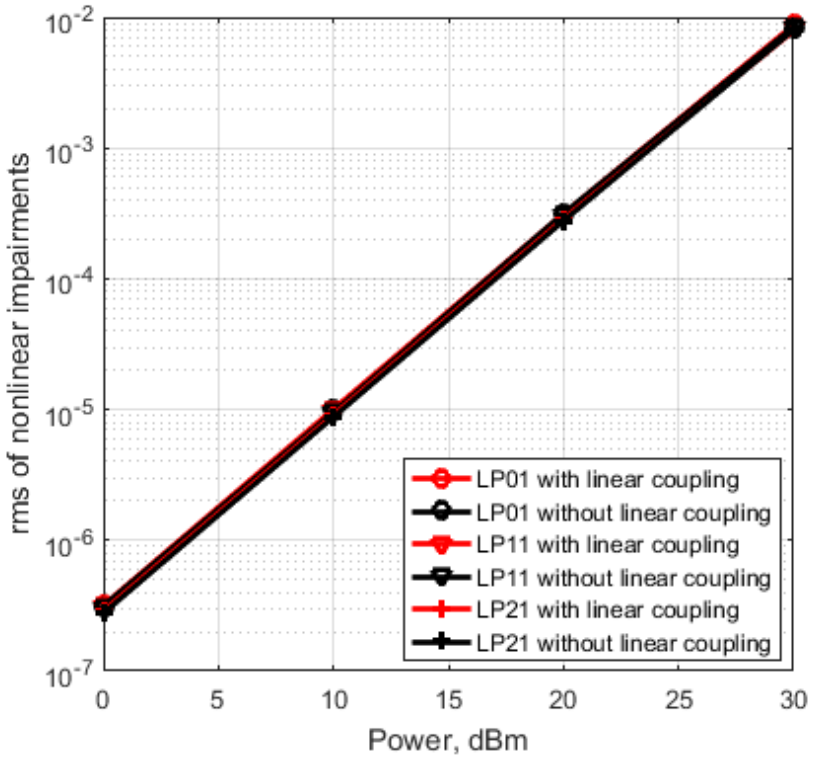

Figure 3. Nonlinear distortions in mode channels in strong-coupling regime with $5 \mathrm{~m}$ mean length coupling sections at 1550 nm. a) QAM-16; b) QAM-64.

Presented results show that strong linear mode coupling lead to the increase of nonlinear impairments in all mode channels for both types of fiber. Comparing it with [19] one can see that in the case of QAM modulation difference between nonlinear impairments in various spatial modes are sufficiently less than in the case of OOK modulation. Moreover, increasing of nonlinear impairments caused by linear coupling in weak-coupling regime for QAM-modulation is less pronounced than for OOK. Thus, QAM-modulated signal is more robust for increasing of nonlinear impairments in fibers with mode coupling than OOK signal. 


\section{ACKNOWLEDGEMENTS}

This work is partially supported by the Ministry of Education and Science of Russian Federation and the Council by grants of the President of Russian Federation under the State support for young scientists.

\section{REFERENCES}

[1] Sakaguchi, J., "Space division multiplexed transmission of 109-Tb/s Data Signals Using Homogeneous SevenCore Fiber," J. of Light w. Technol. 30(4), 658-665 (2012).

[2] Wang, J. et al., "Multimode communications using orbital angular momentum," Optical Fiber Telecommunications VIB: Systems and Networks, Kaminow, I. P., Li, T., Willner, A. E., Eds., Elsevier (2013).

[3] Llorente, R., Morant, M., Garcia-Rodriguez, D., Corral, J. L., "Spatial division multiplexing in the short and medium range: From the datacenter to the fronthaul," 19th International Conference on Transparent Optical Networks, 1-4 (2017).

[4] Fiorani, M., Tornatore, M., Chen, J., Wosinska, L., Mukherjee, B., "Spatial division multiplexing for high capacity optical interconnects in modular data centers," IEEE/OSA Journal of Optical Communications and Networking 9(2), A143-A153 (2017).

[5] Nakajima, K., Matsui, T., Saito, K., Sakamoto, T., Araki, N., "Multi-Core Fiber Technology: Next Generation Optical Communication Strategy," IEEE Communications Standards Magazine 1(3), 38-45 (2017).

[6] Bozinovic, N., Kristensen, P., Ramachandran, S., "Are orbital angular momentum (OAM/vortex) states of light long-lived in fibers?," Frontiers Optics OSA Tech. Dig. (CD), paper LWL3 (2011).

[7] Bozinovic, N., Yue, Y., Ren, Y., Tur, M., Kristensen, P., Willner, A. E., Ramachandran S., "Orbital angular momentum (OAM) based mode division multiplexing (MDM) over a Km-length fiber," in European Conference and Exhibition on Optical Communication, OSA Technical Digest (online), paper Th.3.C.6 (2012).

[8] Bozinovic, N., Yue, Y., Ren, Y., Tur, M., Kristensen, P., Huang, H., Willner A. E., "Terabit-scale orbital angular momentum mode division multiplexing in fibers," Science 340, 1545-1548 (2013).

[9] Ho, K.-P., Kahn, J. M., "Mode coupling and its Impact on spatially multiplexed systems," Optical Fiber Telecommunications VIB: Systems and Networks, Kaminow, I. P., Li, T., Willner, A. E., Eds. Elsevier (2013).

[10] Winzer, P. J., Ryf, R., Randel, S., "Spatial multiplexing using multiple-input multiple-output signal processing," Optical Fiber Telecommunications VIB: Systems and Networks, Kaminow, I. P., Li, T., Willner, A. E., Eds. Elsevier (2013).

[11] Van Uden, R. G. H., Okonkwo, Ch. M., Sleiffer, V. A. J. M., Waardt, de, H., Koonen, A. M. J., "MIMO equalization with adaptive step size for few-mode fiber transmission systems," Opt. Express 22(1), 119-126 (2014).

[12] Lyubopytov V. S. et al., "Optical-domain Compensation for Coupling between Optical Fiber Conjugate Vortex Modes," Conference on Lasers and Electro-Optics Pacific Rim, Paper PDP_T12_1001 (2015).

[13] Poletti, F., Horak, P., "Description of ultrashort pulse propagation in multimode optical fibers," J. Opt. Soc. Am. B. 25(10), 1645-1654 (2008).

[14] Mumtaz, S., Essiambre, R.-J., Agrawal, G. P., "Nonlinear Propagation in Multimode and Multicore Fibers: Generalization of the Manakov Equations," J Lightwave Technol. 31(3), 398-406 (2013).

[15] Antonelli, C., Shtaif, M., Mecozzi, A., "Modeling of Nonlinear Propagation in Space-Division Multiplexed Fiber-Optic Transmission," J Lightwave Technol. 34(1), 36-54 (2016).

[16] Antonelli, C., Golani, O., Shtaif, M., Mecozzi, A., "Nonlinear interference noise in space-division multiplexed transmission through optical fibers," Opt. Express 25(12), 13055 - 13078 (2017).

[17] Khosravani, R., Song, Y. W., Xie, Y., Yan, L.-S., Willner, A. E., Menyuk C. R., "Bit-pattern-dependent polarization rotation in first-order PMD-compensated WDM systems," Optics Comm. 257(1), 191-196 (2006).

[18] Kutluyarov, R. V., Lyubopytov, V. S., Bagmanov, V. Kh., Sultanov, A. Kh., "Influence of the linear mode coupling on the nonlinear impairments in few-mode fibers," Procedia Engineering 201C, 148-154 (2017).

[19] Kutluyarov, R. V., Bagmanov, V. Kh., Antonov, V. V., Sultanov, A. Kh., Lyubopytov, V. S., "Increase of nonlinear signal distortions due to linear mode coupling in space division multiplexed systems," International Multi-Conference on Engineering, Computer and Information Sciences (SIBIRCON), 282-286 (2017). 
[20] Lyubopytov, V. S., Kutluyarov, R. V., Bagmanov, V. Kh., Sultanov, A. Kh., "Method for determination of the principal modes in a few-mode optical fiber using a multibranch DOE," (in Russian), Computer Optics 38 (4), 727-736 (2014).

[21] Agrawal, G. P., [Nonlinear Fiber Optics, 4 ed.] Academic, San Diego, CA, (2007). 